\title{
La localización de las estaciones de Alta Velocidad en España
}

\author{
Carmen Bellet SANFELIU \\ Departamento de Geografía y Sociología \\ Universitat de Lleida \\ c.bellet@geosoc.udl.cat \\ Joan JURADO ROTA \\ Departamento de Geografía y Sociología \\ Universitat de Lleida \\ jjurado@geosoc.udl.cat
}

Recibido: 18 de marzo del 2014

Enviado a evaluar: 8 de mayo del 2014

Aceptado: 24 de junio del 2014

\section{RESUMEN}

La expansión de la red de alta velocidad ferroviaria (AVF) española ha comportado la combinación de lógicas diferentes de implantación. A continuación se analizan las tipologías de la localización de las estaciones teniendo en cuenta su inserción en el tejido urbano y su relación con el conjunto del sistema ferroviario. La lógica de eficacia económica en la que se basa el modelo español y que privilegia las relaciones entre las grandes metrópolis explica que la localización de la estación en éstas suela ser central o tangente, mientras que la localización periférica suele responder a espacios intermedios y menos densamente poblados. Las estaciones se inscriben en sistemas territoriales complejos en los que la localización tiende a una optimización triple: la de la velocidad, la del servicio y la de sus potencialidades.

Palabras clave: Alta velocidad ferroviaria, estación de ferrocarril, infraestructuras de transporte

\section{Location of high speed-rail station in Spain}

\begin{abstract}
The expansion of the Spanish high-speed rail (HSR) network has endured the combination of different logics of implantation. In the text there are analyzed the typologies of HSR station location bearing in mind his insertion in the urban structure and his relation with the set of the railway system. The logic of economic efficiency, which is on the base of the Spanish HSR system, favors the relationship between big metropolises. As a result the station location in these urban spaces tends to be central or tangent. Meanwhile peripheral location ones are usually the case of intermediate spaces and less densely populated. The stations register in complex territorial systems in which station location tends to answer a triple optimization: that of the speed, that of the service and that of his potentials.
\end{abstract}

Key words: High speed-rail, rail station, transport infrastructure 


\section{La localisation des gares ferroviaires à grande vitesse en Espagne}

\section{RÉSUMÉ}

L'expansion du réseau de la grande vitesse espagnole (AVF) a comporté une combinaison d'implantations différentes. Voici l'analyse de la localisation des gares en tenant compte de leur insertion dans le tissu urbain et de leur relation avec l'ensemble du système ferroviaire. Pour comprendre comment la localisation d'une gare devient centrale ou tangente, on suit la logique de l'efficacité économique sur laquelle est basé le modèle espagnol et qui favorise les relations entre les grandes métropoles, alors que la localisation périphérique répond habituellement à des espaces intermédiaires et moins densément peuplés. Les gares s'inscrivent dans des systèmes territoriaux complexes où la localisation est dirigée vers une optimisation triple: celle de la vitesse, celle du service et celle des potentialités.

Mots clé: Grande vitesse ferroviaire, gare, infrastructures de transport

\section{INTRODUCCIÓN ${ }^{1}$}

Para sacar el máximo aprovechamiento de la infraestructura la alta velocidad ferroviaria (AVF) trata de buscar un equilibrio entre velocidad y servicio. Cuando prima la primera suele perjudicar a los servicios intermedios realizando menos paradas. Cuando prima la segunda se perjudica el máximo desarrollo de la velocidad. En esa relación deviene central el papel de la localización de la estación que se encuentra en el centro de ese delicado equilibrio más cuando consideramos que la estación se inscribe en un espacio que no es neutro y que se constituye, especialmente cuando hablamos de infraestructuras de transporte, en un tercer aspecto a considerar en la ecuación: velocidad, servicio y territorio en el que se inscribe la alta velocidad ferroviaria (Facchinetti-Mannone y Bavoux, 2010).

En el presente texto y siguiendo diversos estudios franceses se plantea una clasificación de las diferentes implantaciones de las estaciones según sea el trazado y su localización respecto a la estructura de los asentamientos en el territorio en el que se implanta la infraestructura. Los trabajos de Zembri, Troin o los de Groupe Ten establecían la clasificación de las estaciones de TGV en Francia con criterios semejantes (Zembri, 2005; Troin, 1997; Facchinetti-Mannone y Bavoux, 2010). El establecimiento de tipologías puede contribuir a la comprensión de los diferentes retos que plantea la cuestión de la localización de la estación y con ello guiar las acciones tanto de planificación territorial como sobre la política y gestión de la misma infraestructura (Zemp et al., 2011). El establecimiento de tipologías o clasificaciones

${ }^{1}$ Este artículo se inscribe dentro del Proyecto de Investigación Fundamental no orientada del Ministerio de Economía y Competitividad. Plan Nacional de I+D+I (213-2015). Ref. CSO2012-34629: "Patrones de transformación urbana y estrategias asociadas a la alta velocidad ferroviaria en España (URBATAV)". Dirigido por la Dra. Carmen Bellet. 
permite identificar de forma concreta cuales son los casos comparables e identificar mejor los retos y las oportunidades según sea el tipo (Díaz Márquez y Ureña, 2010).

Además la integración y la capacidad de dinamización de la AVF dependen en buena medida de la localización y características de la estación (Bellet y Gutiérrez, 2011). Tal y como ya han destacado algunos estudios las estaciones periféricas ofrecen la posibilidad de desarrollar nuevo suelo a su alrededor para generar operaciones de nueva centralidad pero presentan dificultades de integración a la estructura urbana/territorial y necesitan buenas medidas y acciones sobre la accesibilidad y el transporte local (Ribalaygua, 2005; Bellet et al. 2010a).

En este trabajo se considera, en primer lugar, la estación como un sistema espacial complejo, para establecer con mayor entendimiento las limitaciones y oportunidades que entran en juego en el momento de decidir la localización de la estación. A continuación, y en segundo lugar, se desarrolla una tipología de la localización de las estaciones de alta velocidad ferroviaria en España, considerando no sólo la red de alta velocidad ferroviaria, su relación con el ferrocarril convencional y su inserción en la estructura urbana/territorial. Consideramos este un primer paso ineludible para comprender mejor las relaciones que se producen entre la nueva infraestructura y el territorio y diseñar buenas políticas y acciones para corregir las limitaciones y aprovechar mejor las oportunidades.

\section{LA ESTACIÓN COMO SISTEMA ESPACIAL COMPLEJO}

En la implantación de una infraestructura ferroviaria en el territorio no siempre coinciden las exigencias generadas por el sistema ferroviario con aquellas derivadas del sistema urbano/territorial. En las primeras pesa la concepción de la estación como un nodo de transporte mientras que en las segundas también entra en cuestión la visión de la estación como un lugar entrando dichas concepciones en clara contradicción (Bertolini y Spit, 1998). Algunos estudios parecen sugerir que una buena inserción de la infraestructura exigiría que se conjugaran de forma coherente las necesidades de lo ferroviario con las de lo urbano/territorial, pero esto no siempre ocurre así (Santos, 2007; Bellet et al., 2010a). Muestra de ello son la aparición de estaciones periféricas mal conectadas con las infraestructuras locales/territoriales y con difícil articulación a la trama urbana existente e incluso, un caso más extremo, estaciones periféricas que crean nuevas situaciones territoriales, como es el caso de la estación Guadalajara-Yebes a cuyo alrededor se generó un especulativo desarrollo inmobiliario (Ribalaygua, 2005).

La estación puede considerarse como un sistema espacial singular siendo su ubicación el resultado de la convergencia de múltiples elementos que surgen de las características de la organización del espacio al que se integra y sirve, características que a su vez son modificadas por el sistema-estación directamente, en tanto que objeto especializado, e indirectamente por los cambios que introduce en la accesibilidad que aporta. El sistema-estación se inscribe así a su vez en un sistema espacial complejo con el que mantiene múltiples relaciones y que estaría al menos compuesto por tres elementos en interacción: 
- Su localización: la estación es un lugar definido por una posición geográfica con unos atributos específicos relacionados con la situación que ocupa tanto dentro la red ferroviaria (en la que actúa como nodo) como del territorio en el que se inscribe (características del lugar: densidad, accesibilidad, estructura de asentamientos, etc.).

- Una organización espacial específica: en relación con su función de interfaz entre la red y el territorio al que sirve que se expresa a través del arreglo espacial de la misma estación y del entorno en la que se ubica.

- Una explotación funcional variable (gestión de los flujos, gestión de las correspondencias y de la utilización de los diferentes servicios) según las características de los servicios e intensidad de su frecuentación.

La elección de la localización de la estación suele ser el resultado de la valoración entre oportunidades y limitaciones que dicta la organización del espacio. Aunque la misma infraestructura es susceptible a la vez de modificar las estructuras y las dinámicas espaciales existentes (Figura 1). Las restricciones o limitaciones que se barajan en la elección de la localización podrían concretarse en las siguientes:

- Restricciones técnicas impuestas por las mismas características de la infraestructura (línea de alta velocidad) y las modalidades de explotación de la estación (funcionamiento y explotación).

- Restricciones o limitaciones físicas, medioambientales, paisajísticas y normativas ligadas a la inserción espacial de las líneas y estaciones.

- Restricciones y limitaciones financieras: costes de implantación espacial y de explotación de la infraestructura.

Existen fuertes interrelaciones entre estas limitaciones: el coste de la implantación de la infraestructura varía, por ejemplo, en función de la importancia de las restricciones técnicas y espaciales. Sin embrago estas restricciones en la implantación pueden relativizarse en función de las oportunidades que genera la localización. Así por ejemplo un lugar penalizado por las altas limitaciones espaciales puede ser preferible a otro si fortalece la viabilidad comercial de la estación.

Mientras que las posibilidades/oportunidades, capaces incluso de superar las limitaciones anteriormente citadas podrían concretarse en las siguientes (Bellet et al., 2010a):

- La viabilidad económica potencial depende del potencial de clientela del área determinado por el volumen/densidad, la distribución de la población y las actividades y la reducción del tiempo de viaje.

- La accesibilidad en función de la calidad de integración del sistema-estación a las redes de comunicación preexistentes o en proyecto.

- Las oportunidades de desarrollo territorial y dinamización socioeconómica local variables en función de las propias características territoriales y las estrategias desarrolladas por los diferentes agentes que interactúan en el territorio. 
Figura 1. Factores en juego en la decisión de localización de la estación: limitaciones y oportunidades.

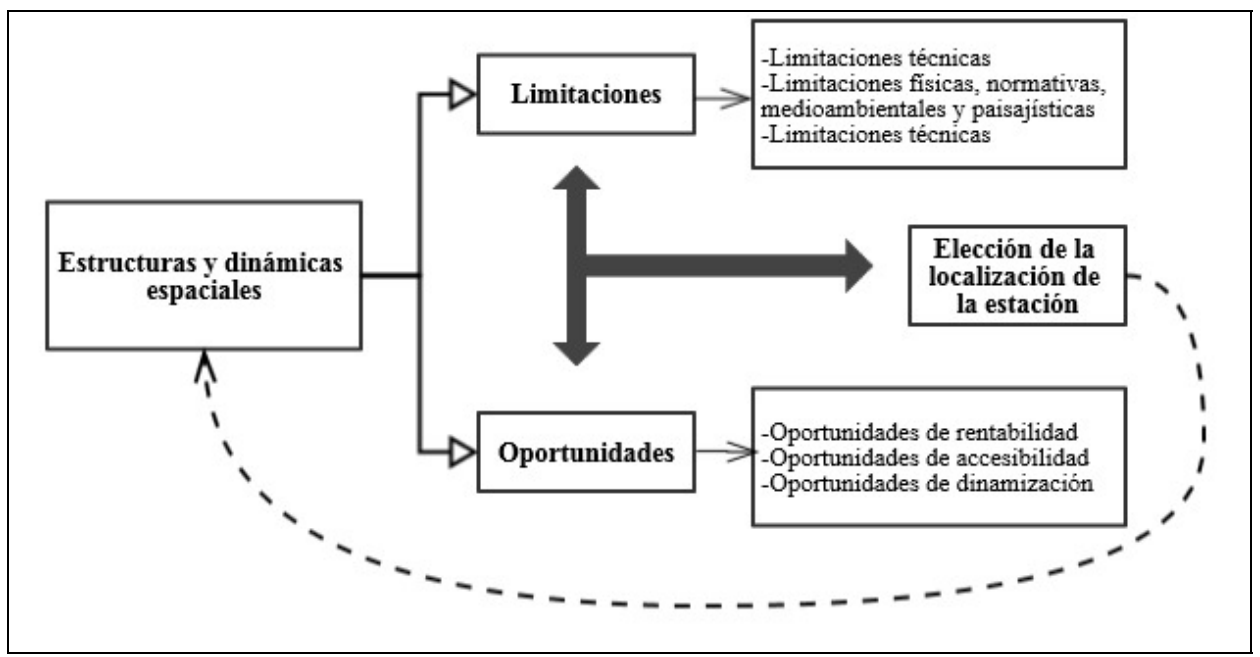

Fuente: Elaboración propia.

La elección de la localización de la estación refleja las diferentes lógicas/visiones de implantación de los agentes que intervienen en el proceso, como ya hemos comentado. Y aunque de entre las opciones suele pesar mucho la lógica ferroviaria (la de la rentabilidad; la de la accesibilidad y eficiencia de los servicios), los cambios contextuales hacen que la estación no pueda ser considerada exclusivamente como un punto de acceso a la red bajo la responsabilidad exclusiva del gestor de la infraestructura y la empresa ferroviaria. Lugar de interconexión de diferentes redes, la estación deviene un polo de intercambios complejo al servicio de los operadores y un polo de servicios cada vez más integrado a lo urbano y la estructura territorial. La estación debe entenderse a la vez como un nodo y un lugar cuyas interacciones son también cambiantes en función de la escala (Bertolini y Spit, 1998).

Así en el momento de la elección de la localización de una nueva estación tres tipos de lógicas estarían en juego reflejando las preocupaciones de los múltiples actores involucrados en el proceso de decisión (ver Tabla 1). 
Tabla 1. Multiescalaridad y lógicas en juego en la localización de las estaciones.

\begin{tabular}{|c|c|c|c|c|}
\hline & $\begin{array}{c}\text { Escala europea y } \\
\text { nacional }\end{array}$ & Escala regional & Escala local & $\begin{array}{c}\text { Escala barrio de la } \\
\text { estación }\end{array}$ \\
\hline $\begin{array}{l}\text { Lógicas de } \\
\text { rentabilidad }\end{array}$ & $\begin{array}{l}\text { Garantizar la } \\
\text { eficiencia de la red } \\
\text { (reducción de los } \\
\text { tiempos) }\end{array}$ & $\begin{array}{l}\text { Ampliar el área de } \\
\text { influencia de la } \\
\text { estación }\end{array}$ & $\begin{array}{l}\text { Captar clientela del } \\
\text { área de influencia }\end{array}$ & $\begin{array}{l}\text { Captar usuarios } \\
\text { diferentes }\end{array}$ \\
\hline $\begin{array}{l}\text { Lógicas del servicio } \\
\text { (eficiencia y } \\
\text { accesibilidad) }\end{array}$ & $\begin{array}{l}\text { Dimensionar la oferta } \\
\text { de servicios } \\
\text { (frecuencias y } \\
\text { destinos) }\end{array}$ & $\begin{array}{l}\text { Asegurar una buena } \\
\text { accesibilidad al } \\
\text { conjunto regional }\end{array}$ & $\begin{array}{l}\text { Integrar la estación a } \\
\text { las diferentes redes de } \\
\text { transporte }\end{array}$ & $\begin{array}{l}\text { Garantizar un } \\
\text { funcionamiento } \\
\text { óptimo del nodo } \\
\text { (nodo de intercambio) }\end{array}$ \\
\hline $\begin{array}{l}\text { Lógicas de } \\
\text { desarrollo } \\
\text { territorial }\end{array}$ & $\begin{array}{l}\text { Reforzar la } \\
\text { atractividad territorial }\end{array}$ & $\begin{array}{l}\text { Integrar la estación en } \\
\text { las estrategias de } \\
\text { desarrollo regional }\end{array}$ & $\begin{array}{l}\text { Integrar la estación en } \\
\text { las dinámicas } \\
\text { espaciales y } \\
\text { estrategias de } \\
\text { desarrollo local }\end{array}$ & $\begin{array}{l}\text { Elegir una } \\
\text { localización y modelo } \\
\text { coherente con los } \\
\text { proyectos locales }\end{array}$ \\
\hline
\end{tabular}

Fuente: Reelaboración propia, a partir de Facchinetti-Mannone, V. 2010.

La lógica de la rentabilidad económica, que trata de controlar los costos sin perjudicar las exigencias comerciales exigiría una localización que, sin penalizar el tiempo, permita drenar hacia la estación un alto número de clientes potenciales.

La lógica del servicio trata de sacar provecho a la nueva accesibilidad. Se trata desde esta lógica de determinar la ubicación que ofrezca a la población y a los principales generadores de tráfico en los territorios en cuestión, la accesibilidad más eficiente a las diferentes redes en términos de dimensionamiento de la oferta de AVF, de tiempo de acceso y de intermodalidad.

Por último, la lógica del desarrollo territorial opta por una ubicación que favorezca la mejor integración posible de la estación en la dinámica espacial y sea coherente con los proyectos locales en marcha para aprovechar la atractividad atribuida a la nueva estación. La lógica del desarrollo refleja las preocupaciones de las autoridades locales que ansiosas por aprovechar la accesibilidad proporcionada por la estación ponen en práctica estrategias de valoración específica (Bellet et al., 2010a).

\section{CARACTERIZACIÓN DE LA AVF ESPAÑOLA Y LA CUESTIÓN DE LA LOCALIZACIÓN DE LAS ESTACIONES}

La introducción de la AVF en España se produce el 21 de abril de 1992 con la inauguración del tramo Madrid-Sevilla $(471 \mathrm{~km})$ en el corredor sur. Este primer tramo pone en servicio cuatro estaciones. Posteriormente, la red de AVF ha ido siendo ampliada de forma notable, especialmente a partir del año 2003 (ver Tabla 2). El desarrollo de otros tres ejes estratégicos de Alta Velocidad: Madrid-Barcelonafrontera francesa; Madrid-Levante; y Madrid-noroeste peninsular (inconcluso), más la conexión Córdoba-Málaga y Madrid-Toledo, ha permitido alcanzar los cerca de 3.000 kilómetros de Líneas de Alta Velocidad (LAV), permitiendo conectar 30 ciudades a 
través de 31 estaciones que darían servicio al $60 \%$ de la población española (según datos del INE de 2012).

Figura 2. Factores en juego en la decisión de localización de la estación: limitaciones y oportunidades.

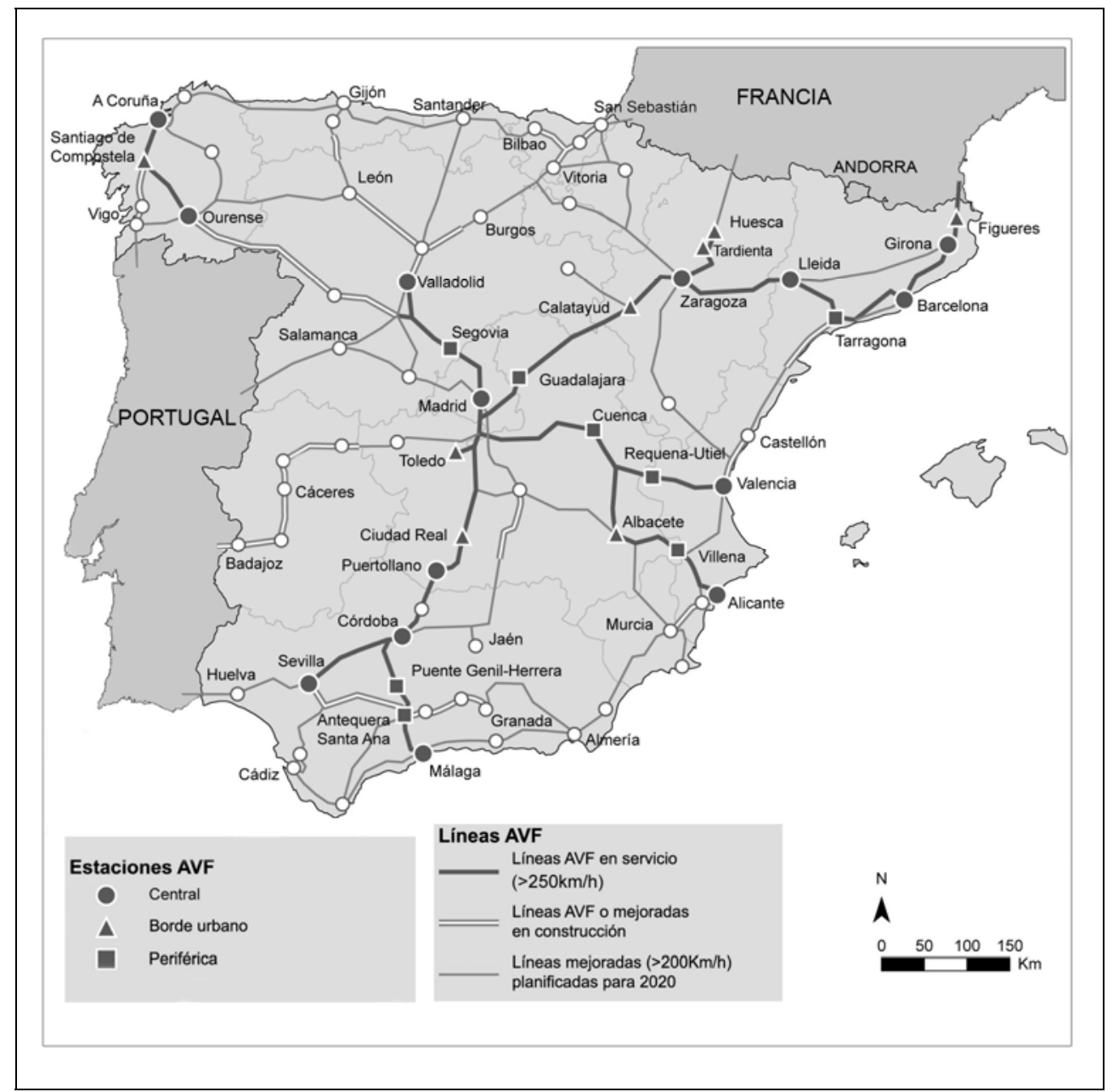

Fuente: Elaboración propia. 
Cabe señalar además que a través de los cambiadores de ancho instalados en la red convencional se benefician actualmente de los servicios de Alta Velocidad Ferroviaria (AVF) otras 50 ciudades del país (Figura 2), tal y como constaba inicialmente en el PEIT con horizonte 2020. Los ya más de 20 años de experiencia en el país con el AVF y las expectativas que todavía genera a escala local invitan a una reflexión global sobre cómo se ha integrado la nueva infraestructura en el medio urbano y cómo se pueden poner en valor los cambios que ésta suscita. Cuestiones en las que la localización de la estación resulta un asunto primordial.

Además existen ciertas características de la red de ferrocarril de alta velocidad española que deben tenerse presentes de partida. En primer lugar debemos recordar que en España, como en Portugal, las redes estatales convencionales de ferrocarriles disponen de un ancho diferente (1,688 metros), mientras que la nueva red de alta velocidad se ha construido de forma independiente en ancho UIC. Tan solo algunos trenes pueden interoperar con los diferentes anchos con lo que se pierde el efecto de la red de ferrocarril completa. Un esquema de red independiente para la alta velocidad ferroviaria que reproduce la clásica radialidad de las infraestructuras en la península ibérica con centro en Madrid. Sobre la red de alta velocidad se ha construido estaciones cada 60-70 km. Como ya ocurrió en Francia y en Alemania los primeros planteamientos sobre la configuración de la alta velocidad pasaron de los criterios funcionales de la línea (unir de forma eficaz dos puntos distantes entre si) a criterios más relacionados con el desarrollo territorial, tratando de incorporar espacios intermedios en la red. Los criterios "territoriales" facilitaron así la incorporación de más nodos en las líneas incorporando la conexión para territorios menos densos y /o articulados por ciudades medias y pequeñas. Aunque claro está la lógica de la eficiencia económica acabaría imponiéndose en la localización tangente o periférica de algunas paradas intermedias, como veremos en el siguiente apartado.

Así pues, las dificultades en la interoperabilidad de las redes convierten el trazado y la localización de la estación en una cuestión central y clave en el caso español (Guirao, 2013; Bellet y Gutiérrez, 2011). Las estaciones, los nodos de las líneas, se convierten no sólo en los puntos de acceso al sistema sino también en lugares singulares llamados a tener un papel relevante en la nueva configuración urbana/territorial, pero también, a condicionar el papel de la AVF en la ciudad/territorio en la que se implanta. Cuestiones como la ubicación de la estación, la accesibilidad local en transporte privado y público o las actividades del entorno, serán factores que terminarán por condicionar al papel de la AVF en cada localidad (Bertolini y Spit, 1998; Van den Berg y Pol, 1998).

Las limitaciones de la red y los servicios del ferrocarril convencional en España ayudan también a entender las grandes expectativas de dinamización que a escala local se generaban entorno la llegada del tren de alta velocidad. Las ciudades y territorios luchaban para quedar articuladas a la nueva red de ferrocarril que se asociaba de forma mecánica a un instrumento de desarrollo territorial. Un ejemplo de ello fue el caso de Segovia que obtuvo la estación tras arduos debates y demandas territoriales (Bellet et al., 2010b). 
Tabla 2. Cronología de la Alta Velocidad Ferroviaria en España.

\begin{tabular}{|l|l|l|}
\hline \multicolumn{1}{|c|}{ Año } & \multicolumn{1}{|c|}{ Línea } & \multicolumn{1}{c|}{ Estaciones } \\
\hline Abr.1992 & LAV Madrid-Sevilla & $\begin{array}{l}\text { Madrid Atocha, Ciudad Real, Puertollano, Córdoba, } \\
\text { Sevilla }\end{array}$ \\
\hline Oct.2003 & $\begin{array}{l}\text { LAV Madrid - Barcelona - Frontera } \\
\text { Francesa }\end{array}$ & $\begin{array}{l}\text { Guadalajara-Yebes, Calatayud, Zaragoza-Delicias, } \\
\text { Lleida-Pirineus }\end{array}$ \\
\hline Nov. 2003 & LAV Zaragoza-Huesca & Huesca, Tardienta \\
\hline Nov. 2005 & LAV Madrid-Toledo & Toledo \\
\hline Dic. 2006 & $\begin{array}{l}\text { LAV Madrid - Barcelona - Frontera } \\
\text { Francesa }\end{array}$ & Camp de Tarragona \\
\hline Dic. 2006 & LAV Córdoba-Málaga & Antequera Santa Ana, Puente Genil \\
\hline Dic. 2007 & LAV Madrid-Valladolid & Madrid-Chamartín, Segovia-Guiomar, Valladolid \\
\hline Dic. 2007 & LAV Córdoba-Málaga & Málaga-María Zambrano \\
\hline Feb. 2008 & $\begin{array}{l}\text { LAV Madrid - Barcelona - Frontera } \\
\text { Francesa }\end{array}$ & Barcelona-Sants \\
\hline Dic. 2010 & $\begin{array}{l}\text { LAV Madrid - Barcelona - Frontera } \\
\text { Francesa }\end{array}$ & Figueres \\
\hline Dic. 2010 & LAV Madrid-Valencia-Murcia & Cuenca, Albacete, Requena-Utiel, Valencia \\
\hline Dic. 2011 & LAV Madrid-Galicia-Eje Atlántico & Ourense, Santiago, A Coruña \\
\hline Ene. 2013 & $\begin{array}{l}\text { LAV Madrid - Barcelona - Frontera } \\
\text { Francesa }\end{array}$ & Girona \\
\hline Jun. 2013 & LAV Madrid-Valencia-Murcia & Villena, Alicante \\
\hline
\end{tabular}

Fuente: Elaboración propia, a partir ADIF

En principio, la vocación de la AVF es la de enlazar de forma eficiente el centro de las grandes ciudades (Gutiérrez Puebla, 2004). Por ello en estas ciudades, y pese a las costosas inversiones necesarias suele implantarse la infraestructura necesaria en posición central que garantiza un mayor aprovechamiento de la accesibilidad, conectividad y garantiza cierto grado de intermodalidad (Garmendia et al., 2012). Además, y con ello, se potencian (o crean) las centralidades urbanas y se contribuye a la regeneración o redinamización de los barrios del entorno. Para ello suelen remodelarse o adaptarse las infraestructuras ferroviarias con que ya cuentan éstas en dichas posiciones.

La situación es bien diferente en el caso de ciudades medias o pequeñas. Aquí, las fuertes inversiones que acarrearían las posiciones centrales y los minutos que pueden perderse en el trayecto entre las grandes ciudades explican que, en estos casos, la localización de la estación tienda a ser periférica o tangente (Ribalaygua, 2005). En Francia esta es la situación para buena parte de las estaciones TGV que cruzan territorios con poca densidad demográfica (Facchinetti-Mannone, 2010). Y siendo el caso español en cierto sentido parecido al francés, la práctica totalidad de las estaciones de AVF de ciudades medias suelen ser centrales o tangentes, como muestra el mapa de la Figura 1. Ello es en buena parte debido a las fuertes presiones que los agentes locales ejercieron durante los debates sobre las alternativas de los trazados viendo en la obtención de la estación de AVF una fuente de desarrollo local (Lleida, Girona, Albacete, Córdoba, etc.). Ciertamente la construcción de trazados exteriores y 
la localización de estaciones tangentes o periféricas son opciones menos complejas y menos costosas. Pero requieren de proyectos e inversiones paralelas para garantizar la accesibilidad y conectividad territorial de la estación. Además y como ya demostraron en su día algunos casos franceses (Mâcon-Loche, Valence, Vendôme, entre otros) no es tan sencillo aprovechar el suelo disponible en el entorno de estas estaciones periféricas para generar nuevas centralidades. Disponibilidad de suelo y accesibilidad parecen no ser condiciones suficientes para generar nuevas centralidades.

\section{TIPOLOGÍAS DE IMPLANTACIÓN DE ESTACIONES DE AVF EN ESPAÑA}

El esquema que sigue (Figura 3) presenta una clasificación de las estaciones de alta velocidad españolas según sea el trazado y localización de los servicios de AVF, su relación con la red de ferrocarril convencional y su relación con la estructura de asentamientos (núcleos urbanos más importantes) del área en la que se ubica. En esta clasificación se incluyen tan solo las estaciones sobre líneas AVF ya en funcionamiento.

El análisis de la relación entre la elección de la ubicación de la estación y su relación con la organización del espacio se utiliza para especificar las ventajas y desventajas de los distintos tipos de localización con respecto a las cuestiones de rentabilidad, de accesibilidad y de posible capacidad de desarrollo regional. Aunque tan solo a través del estudio detallado de cada uno de los casos pueda llegar realmente a entenderse cómo se tomaron en cuenta estos aspectos en el proceso de decisión. En principio las estaciones estudiadas se pueden agrupar en tres grandes tipos principales de localización: centrales, tangentes o de borde urbano, y, periféricas.

En la localización central de los servicios y estaciones AVF pesarían más las oportunidades que genera la densidad urbana pese a los costos y las limitaciones técnicas y financieras derivadas de su implantación. La ubicación central presenta muchas ventajas (Groupe Ten, 1993):

- El servicio a través del centro proporciona un mayor potencial de clientela lo que repercute en su viabilidad económica.

- Mayor potencial de accesibilidad e integración del nodo a los demás modos de transporte.

- Gran potencial de valoración y dinamización del lugar y entorno a través de actuaciones urbanísticas alrededor de la estación o en el barrio de la estación. $\mathrm{La}$ implantación de la AVF en el medio urbano ha sido leída en España como una oportunidad histórica para desarrollar importantes transformaciones urbanísticas y reinterpretar la difícil relación entre el tren y la ciudad. La implantación central de la AVF ha conllevado el soterramiento de buena parte de la travesía ferroviaria, y en buena parte de los casos, el desplazamiento de grandes piezas ferroviarias al exterior: talleres, áreas de mercancías y almacenaje, etc. Con ello se han liberado importantes paquetes de suelo en posición más o menos central dando lugar a operaciones de renovación y reestructuración urbanística más o menos importantes (Bellet y Gutiérrez, 2011). 
Figura 3. Tipos de estación de AVF con localización central en España.

\begin{tabular}{|c|c|c|c|}
\hline \multicolumn{4}{|c|}{ TIPO 1: ESTACIONES CENTRALES } \\
\hline $\begin{array}{l}\text { 1a: LAV y estación central en } \\
\text { ramal urbano con línea } \\
\text { convencional }\end{array}$ & $\begin{array}{l}\text { 1b: Estación central en ramal } \\
\text { LAV terminal }\end{array}$ & $\begin{array}{c}\text { 1c: LAV y convencional en } \\
\text { travesía urbana. Estación } \\
\text { central }\end{array}$ & $\begin{array}{c}\text { 1d: Estación cental con LAV } \\
\text { terminal y línea convencional } \\
\text { en travesia }\end{array}$ \\
\hline $\begin{array}{l}\text { Madrid-Atocha (1992) } \\
\text { Zaragoza-Delicias (2003) } \\
\text { Lleida (2003) } \\
\text { Valladolide (2007) } \\
\text { Ourense (2011) }\end{array}$ & Toledo (2005) & $\begin{array}{c}\text { Puertollano (1992) } \\
\text { Córdoba (1992) } \\
\text { Sevilla (1992) } \\
\text { Barcelona-Sants (2008) } \\
\text { Girona (2013) } \\
\text { Madrid-Chamartin (2007) }\end{array}$ & $\begin{array}{l}\text { Huesca (2003) } \\
\text { A Coruña (2011) } \\
\text { Malaga (2007) } \\
\text { Valencia (2010) } \\
\text { Alicante }(2013)\end{array}$ \\
\hline \multicolumn{4}{|c|}{ Linea de Alta Velocidad } \\
\hline & Núcleo urbano & \multicolumn{2}{|c|}{ Linea de Ferrocarril convencional } \\
\hline
\end{tabular}

Fuente: Elaboración propia, a partir de proyectos de implantación y documentos de ADIF.

El aprovechamiento de estas potencialidades, basadas en la densidad y centralidad, compensarían las limitaciones derivadas de la densidad urbana y centralidad del lugar:

- Penaliza los tiempos de trayecto y parada y aumenta las limitaciones de la explotación.

- Aumento de los costes financieros y dificultades técnicas para la implantación central de los servicios bien sea con la remodelación de la estación clásica para coger los nuevos servicios o la construcción de una estación nueva.

- Posibles penalizaciones en la accesibilidad por la densidad de tráficos en el entorno de la estación.

En general en España, y según se muestra en las tipologías, la localización central prima en las grandes ciudades que disponen de estaciones de tipo intermodal: Barcelona-Sants, Madrid Atocha y Chamartín, Sevilla, Zaragoza o Valencia. Sin embargo, la localización central, en buena parte integrando los servicios a la estación tradicional, ha sido también la opción de buena parte de ciudades medias y capitales de provincia: Córdoba, Lleida, Ourense, Toledo o Girona.

El segundo de los casos es de la localización de la estación y servicios de AVF en situación tangencial o de borde urbano (Figura 4). Este tipo de localización combina las ventajas de la densidad y centralidad urbana reduciendo las limitaciones a que hemos hecho referencia. En Calatayud y Albacete se aprovecharon las clásicas 
infraestructuras ferroviarias para acoger la AVF. Interesantes son los casos de Ciudad Real y Figueres dónde la implantación de la AVF se produce a través del desmantelamiento de infraestructuras centrales y la construcción de una nueva línea exterior en posición tangente dónde se localiza la nueva estación para acoger tanto los servicios convencionales como de los de AVF.

En España muchas de las localizaciones de las estaciones de AVF son centrales y/o tangenciales, no solo en el caso de metrópolis y grandes áreas urbanas sino también en el caso de muchas ciudades medias y pequeñas. En otros estudios ya se ha relacionado este factor con el tema inmobiliario ya que los proyectos de implantación del nuevo tren coincidieron en el tiempo con el empuje del sector de la construcción y de lo inmobiliario en España. Las plusvalías generadas en los procesos de remodelación y nueva calificación de suelo fueron o esperan ser (según el caso) utilizadas para sufragar los gastos de la implantación del nuevo tren y los proyectos urbanos asociados a su llegada (Bellet y Gutiérrez, 2011). Este sería el caso del proyecto bastante avanzado de remodelación del entorno de la estación en Lleida, el incipiente de Málaga o Alicante o el casi no ejecutado proyecto de Ourense. En todo caso muchos de estos proyectos van a tener que revisarse.

Figura 4. Tipos de estación de AVF con localización tangente o de borde urbano en España.

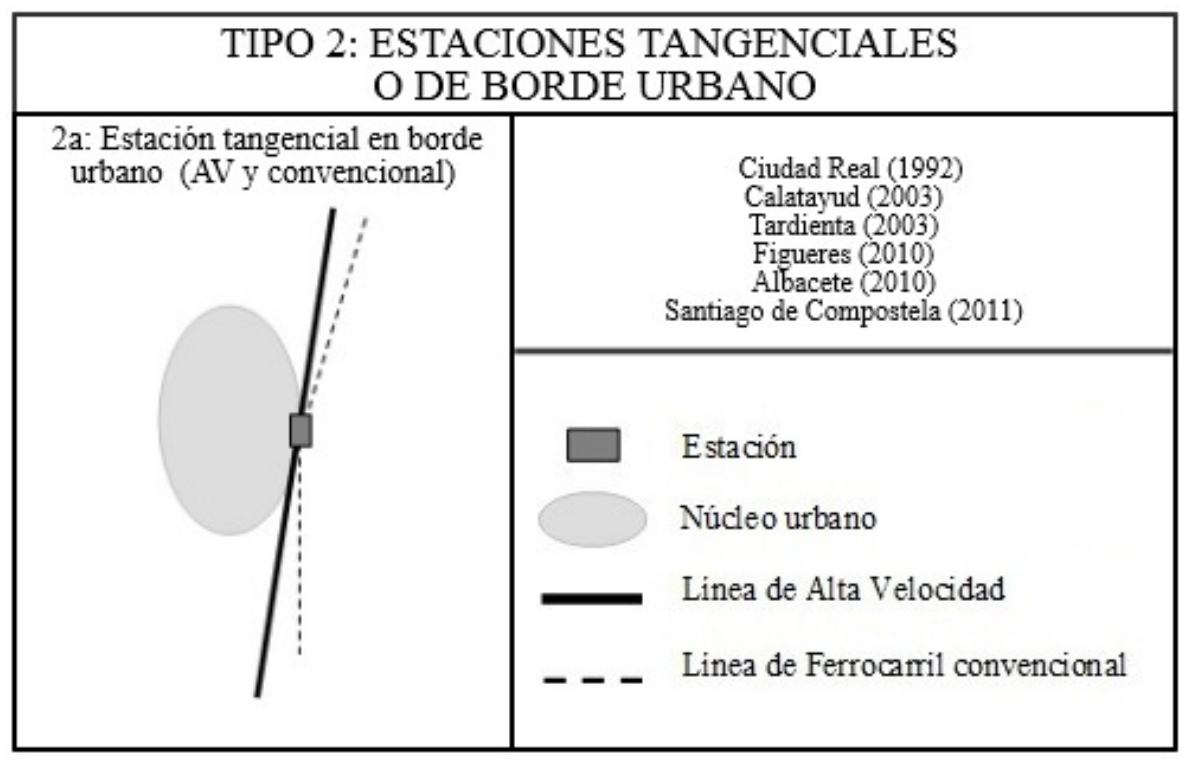

Fuente: Elaboración propia, a partir de proyectos de implantación y documentos de ADIF. 
En caso de localización central o tangente de las estaciones la transformación del sistema ferroviario trata de adaptar su lógica a la estructura urbana existente. Las visiones entre lo ferroviario y la territorial se encuentran más equilibradas. Pero en otros casos encontramos una situación totalmente diferente siendo el sistema ferroviario el que impone su lógica al sistema territorial. Este es el caso de las estaciones periféricas (Figura 5). La dotación de una adecuada accesibilidad y su integración a la estructura urbana/territorial preexistente son los mayores retos que deben asumir este tipo de localizaciones. Aunque en algunos casos se ha leído como una buena ocasión para generar nuevas centralidades (en el caso por ejemplo de estaciones francesas) y nuevos desarrollos de suelo (en el caso de Báldelas en Guadalajara-Yebes por ejemplo).

Figura 5. Tipos de estación de AVF con localización periférica en España.

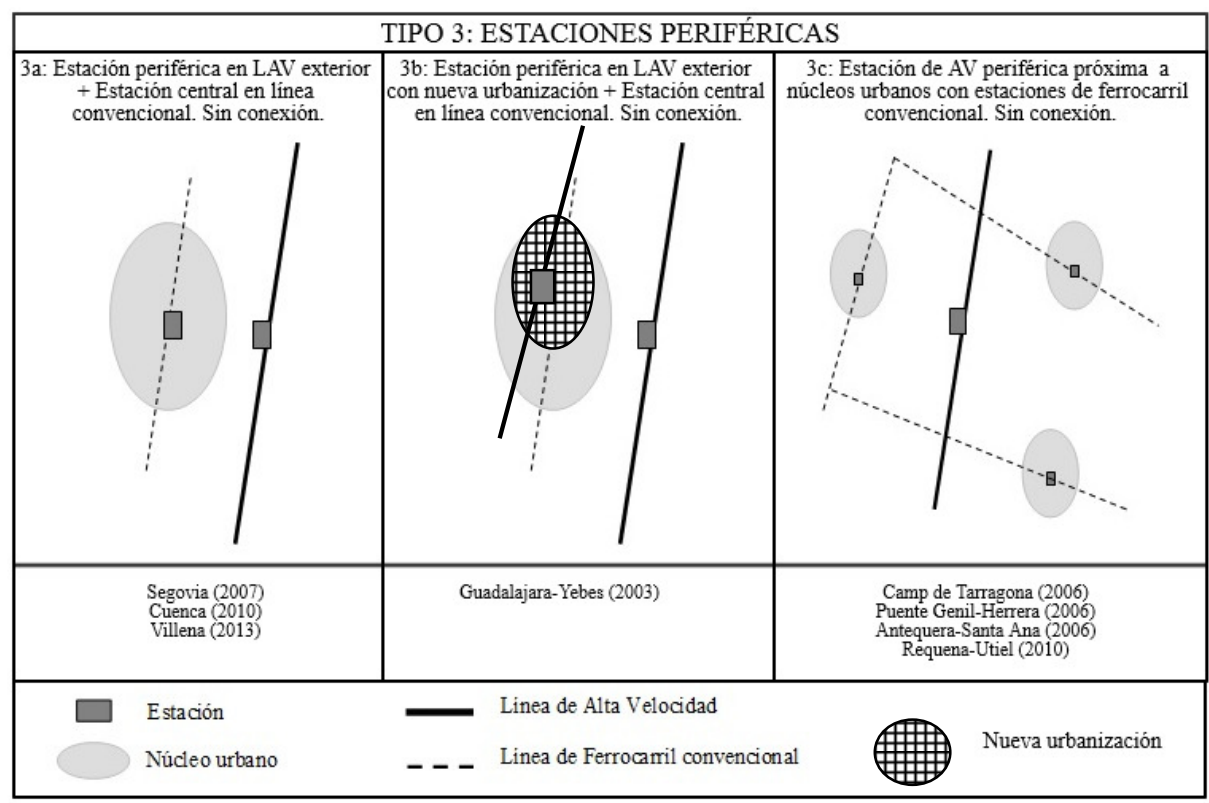

Fuente: Elaboración propia, a partir de proyectos de implantación y documentos de ADIF.

Sin embargo, cabe destacar que existen importantes diferencias entre las estaciones periféricas con que cuenta la AVF en España:

- Una situación habitual es el llamado por los franceses "estaciones-bis" en el que se construye una nueva estación y línea de AVF no vinculada a la red convencional y a una distancia de entre unos 5-10 km., según el caso, del núcleo urbano principal. Este es el caso de Cuenca, Guadalajara, Segovia y Villena. 
- Otra modalidad sería la de estación periférica en territorios rurales de baja densidad, y sin polaridades urbanas claras. Aquí encontraríamos las estacionesbajador de: Antequera-Santa Ana, Puente Genil-Herrera y Requena-Utiel.

- La tercera modalidad es la existente en el Camp de Tarragona dónde a la estación periférica ya existente, en el trazado de la LAV Madrid-Barcelona y a 10-15 km respectivamente de Tarragona y Reus, se le va a sumar una nueva estación periférica en el aeropuerto de Reus y sobre la línea del corredor del Mediterráneo. Una situación única en Europa que plantea importantes problemas de funcionalidad, conectividad y accesibilidad.

\section{CONCLUSIONES}

La elección de la ubicación de las estaciones refleja las diferentes lógicas de implantación de los agentes que intervienen en el proceso. Entre las opciones suele pesar mucho la lógica de la eficiencia, la lógica prevalente en la visión del gestor y operador ferroviarios, aunque los cambios contextuales hacen que la estación no pueda ser considerada exclusivamente como un punto de acceso a la red. Lugar de interconexión de diferentes redes, la estación deviene un polo de intercambios complejo al servicio de los operadores y un polo de servicios cada vez más integrado a lo urbano. La estación deviene así a la vez un nodo y un lugar, un sistema especial complejo y dónde convergen dinámicas multiescalares.

El sistema-estación se inscribe a su vez en un sistema espacial con el que mantiene múltiples relaciones. La ubicación y las características específicas de la estación dependen en gran medida de las características de la organización del espacio en el que se inserta, características que a su vez son modificadas por el sistema-estación.

El análisis de la relación entre la elección de la ubicación de la estación y las características de la organización del espacio se utiliza en el texto para especificar las ventajas y desventajas de los distintos tipos de localización con respecto a las cuestiones de rentabilidad, de accesibilidad y de capacidad de dinamización territorial. Aunque sólo con el estudio detallado de los casos pueda llegar a entenderse cómo se tomaron en cuenta estos aspectos en el proceso de decisión. Las estaciones estudiadas se pueden agrupar en tres grandes tipos principales de localización: centrales, tangentes o de borde urbano, y, periféricas.

Las localizaciones centrales aprovechan sobretodo la densidad urbana y los beneficios que genera la centralidad sobre la accesibilidad. Estas pueden compensar los sobrecostes financieros y las dificultades técnicas que genera su implantación y las limitaciones de servicio (tiempo de trayecto y parada). Por su parte las estaciones tangenciales tratan de conjugar de forma más eficaz las ventajas de la densidad y accesibilidad urbana con los menores costes de implantación y servicio. En España muchas de las localizaciones de las estaciones de AVF son centrales y/o tangenciales, no solo en el caso de grandes áreas urbanas sino también en el caso de muchas ciudades medias y pequeñas. En comparación con el caso francés la localización de estaciones periféricas es en España menos frecuente. Las estaciones periféricas ofrecen suelo a su alrededor pero son más difíciles de integrar a la estructura 
urbana/territorial y necesitan mayores intervenciones para garantizar buena accesibilidad y políticas de transporte.

\section{BIBLIOGRAFÍA}

BELLET, C; ALONSO, P. y CASELLAS, A. (2010a). Infraestructuras de transporte y territorio. Los efectos estructurantes de la llegada del tren de alta velocidad en España. Boletín de la Asociación de Geógrafos Españoles, 52, 143-163.

BELLET, C; ALONSO, P. y CASELLAS, A. (2010b). La integración del ferrocarril de alta velocidad en el medio urbano. El caso de Segovia-Guiomar. Anales de Geografía de la Universidad Complutense de Madrid, vol. 30, 1, 9-26.

BELLET, C, y GUTIERREZ, A. (2011). Ciudad y ferrocarril en la España del Siglo XXI. La integración de la Alta Velocidad Ferroviaria en el medio urbano. Boletín de la Asociación de Geógrafos Españoles, 55, 251-279.

BERTOLINI, L. y SPIT, T. (1998). Cities on rails. The redevelopment of railway station areas. London. Spon Press.

DÍAZ MÁRQUEZ, S. y UREÑA, J (2010). El estudio del papel territorial de los intercambiadores de transporte: revision y propuesta metodológica. Boletín de la Asociación de Geógrafos Españoles, 54, 29-55

FACHINETTI-MANNONE, V. (Dir) (2010). L'implantation des gares de la grande vitesse. Analyse comparée des implantations belges, françaises et espagnoles. Proyecto encargado por la SNCF (Gares et conexions), Paris, enero de 2010. No publicado.

FACHINETTI-MANNONE, V y BAVOUX, J.J. (2010). L'implantation des gares TGV en France: tensions interscalaires, jeux d'acteurs et recompositions spatiales. Belgeo,1-2, 9-22.

GARMENDIA, M.; RIBALAYGUA, C. y UREÑA, J. (2012). High speed rail: implication for cities. Cities, 29, S26-S31.

GUIRAO, B (2013). Spain: highs and lows of 20 years of HSR operation. Journal of Transport Geography, 31, 201-206.

GROUPE TEN (Courson, J. / Remond,E. / Jaouen,M.) (1993). Gares TGV et urbanisme. Paris, Société Nationale des Chemins de Fer Français - Ministère de l'équipement, du logement et des transports, 2 vols.

GUTIERREZ, J. (2004). El tren de alta velocidad y sus efectos espaciales. Investigaciones Regionales, 5, 199-221.

RIBALAYGUA, C. (2005). Nuevas estaciones periféricas de alta velocidad ferroviaria: estrategias para su incorporación a las ciudades españolas. Colección Cuadernos de Ingeniería y Territorio, 5. Universidad de Castilla- La Mancha, Ciudad Real.

SANTOS, L. (2007). Urbanismo y ferrocarril. La construcción del espacio ferroviario en las ciudades medias españolas. Madrid, Fundación de los Ferrocarriles Españoles.

TROIN, F. (1997). Les gares T.G.V. et le territoire: débat et enjeu. Annales de Géographie, 593-594, 34-50. 
TROIN, J. (1995). Rail et aménagement du territoire. Aix-en-Provence, Edisud.

VAN DEN BERG, L. y POL, P. (1998). The European high-speed train and urban development. Experiences in fourteen European urban regions. Aldershot, Ashgate.

ZEMBRI, P (2005). El TGV, la red ferroviaria y el territorio en Francia. Ingeniería y Territorio, 70, 12-19.

ZEMP, S.; STAUFFACHER, M.; LANG, D.J. y SCHOLZ, R.W (2011). Classifying railway stations for strategic transport and land use planning: Context matter! Journal of Transport Geography, 19, 670-679. 